\title{
Tri-mode dual level 3-D image compression over medical MRI images
}

\author{
D. J. Ashpin Pabi", P. Aruna and N.Puviarasan \\ Department of Computer Science \& Engineering, Annamalai University, Tamil Nadu, India
}

Received: 16-October-2016; Revised: 15-December-2016; Accepted: 19-December-2016

(C)2017 ACCENTS

\begin{abstract}
Digital image sequence requires huge storage and high bandwidth for transmitting data in uncompressed form of the multimedia communication. Efficient image compression technique is required to meet the acceptable quality. In this paper, an efficient image compression technique is developed based on the intensity value of the pixels. The proposed image compression algorithm contains two levels of compression. Initially the average between the neighboring of a particular pixel is evaluated and it is assigned to the pixels of the original image. This reduces the correlations of the intensity level. The same pixel assignment is extended towards three dimensional forms along rows, column and the diagonals. To retain the quality of the image the compressed image based on such intensity assignment is encoded using the proposed tri-mode encoding scheme. The encoded bits are decoded at the compression decoder. To reveal the efficiency of the proposed method, the obtained results are compared with the other existing methods. The proposed algorithm has been tested in magnetic resonance imaging (MRI) images of the brain. Simulation results show the proposed method gives the best results over the other existing methods.
\end{abstract}

\section{Keywords}

Image compression, Magnetic resonance images, Color images, Histogram processing, Encoding schemes.

\section{Introduction}

Medical centers and hospitals use large amount of digital medical images for different purposes such as surgical and diagnostic plans. Teleradiology is the most advanced part of telemedicine. The radiologists interpret digital medical images while not physically present in the same location. Hence, these medical images need to be transmitted very fast and it required to store with a minimum capacity. Thus, image compression is used to reduce the redundancies in image and represents it in shorter manner to achieve efficient archiving and transmission of images. Image compression is the process of reducing irrelevant and the redundancy of the image data in order to store or transmit data in an efficient form. Image compression minimizes the size in bytes of an image without degrading the quality of an image to an acceptable level. Image compression techniques are broadly categorized into lossy and lossless compression techniques. Lossy image compression generates reconstructed image with some degradation, whereas lossless preserves image information.

\footnotetext{
*Author for correspondence
}

The lossy compression techniques achieve a high compression ratio. The output of the lossless image compressor corresponds to the original image.

Lossless compression techniques cannot be used over large datasets. In picture archiving and communication systems (PACS), Teleradiology and the remote dataset browsing systems, such lossless compression doesn't suit due to the limited transmission bandwidth and the storage constraints.

The following are some of the methods for lossy compression: chroma subsampling, Transform coding and Fractal compression. The techniques involved in lossless image compression are: run-length encoding, area image compression, differential pulse code modulation (dpcm), entropy encoding, adaptive dictionary algorithms, deflation and chain codes. In this paper, a novel image compression algorithm is implemented based on the intensity values of the neighbouring pixels. The assignment of a pixel value based on the intensity value of the neighbouring pixels reduces the correlation of the pixel value, increases the transmission speed and thus minimized the bandwidth required to transfer the image file. 
The remaining of the paper is arranged with the following sections: Section 2 surveys the literatures; section 3 describes the proposed compression technique with the block diagrams and the algorithms. The results and the comparison to the other methods are discussed in section 4 and section 5 concludes the work.

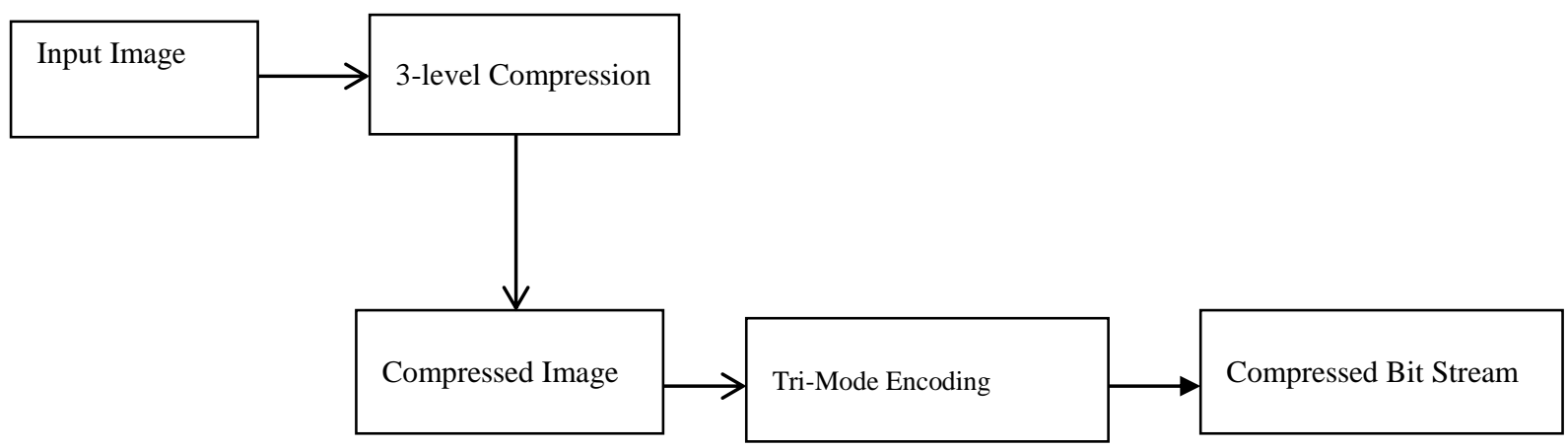

Figure 1 Stages of the proposed compression technique

\section{Overview of related and background work}

There are various image compression techniques available in the literature. Recently there has been abundant interest in transforming based compression methods, including discrete cosine transforms (DCT), discrete wavelet transform (DWT) and more. Moonen et al. (1992) developed a method which packs signal energy into fewer coefficients [1]. Kahu et al. (2013) invented an image compression technique based on the singular value decomposition (SVD). They mainly concentrated to reduce the number of Eigenvalues required to reconstruct an image [2]. Konda et al. (2009) described that the singular value decomposition is a part of a divide and conquer algorithm [3]. The method used by Andrews et al. (1976) shows the application of SVD in digital images; also its importance in image processing and the point spread function representation [4]. Kamm (1998) developed a Newton method which is based on SVD to solve the problems occurs in signal and image processing applications [5]. Yang et al. (1995) combined SVD and vector quantization (VQ) to achieve low bit rate and high quality image coding [6]. $\mathrm{Hu}$ et al. (2014) predicted a single perceptual quality score by selecting local image distortion features using SVD [7]. Gupta et al. (2014) explained vector quantization finds the closest codeword for each image block in the codebook or dictionary [8]. Shih et al. (2012) described an adaptive parametized block based singular value decomposition algorithm [9]. In this paper three mode two level 3-D image compression is introduced to compress the medical images with the acceptable quality loss. Performances of the proposed method are estimated and analysed with state of the art methods such as joint photographic experts group (JPEG) [10], singular value decomposition (SVD) and wavelet difference reduction (WDR) [11].

\section{The proposed compression technique}

Figure 1 shows the stages of the proposed image compression method for medical images. The proposed two level 3-D image compression consists of three stages includes: Initialization, first level compression and the second level encoding. At the initialization stage the input medical MRI brain image is given for compression. The first level compression assigns the intensity of the pixel value based on the neighbouring pixels. Such assignment of the pixel value reduces the correlation of the corresponding pixels, improves the compression ratio and thus minimizes the bits per pixel. The proposed algorithm consists of two main processes: A 3-D image compression and a tri-mode encoding scheme. A block diagram of the proposed compression method is given in Figure 2. The correlation between the pixel values are reduced using the proposed 3-D based compression algorithm. The proposed Trimode encoding scheme reduces the bits assigned to the pixels.

\subsection{Proposed 3-D compression method}

Reducing image correlation between the pixels increases the compression speed of the digital image. The medical MRI brain images are used to learn the performance of the algorithm. The image is divided into $8 \times 8$ blocks. Then, the proposed compression method is applied to each block in order to reduce the file size of the given input image. The proposed image compression algorithm consists of three levels as explained below: 
D. J. Ashpin Pabi et al.

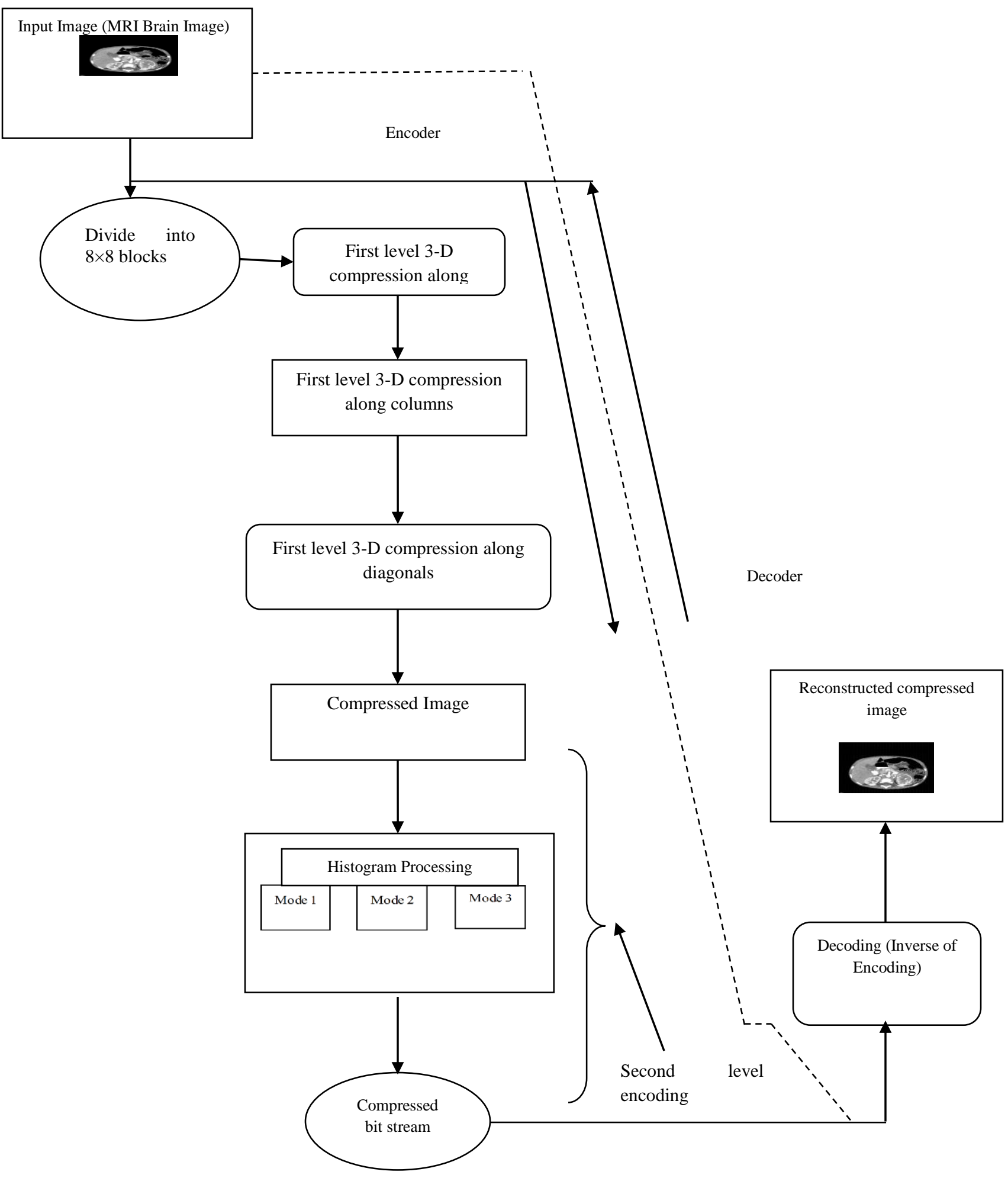

Figure 2 Block diagram of the proposed 3-D compression technique 
Irqut block

Level 1 along rows

Level 2 along ohumms

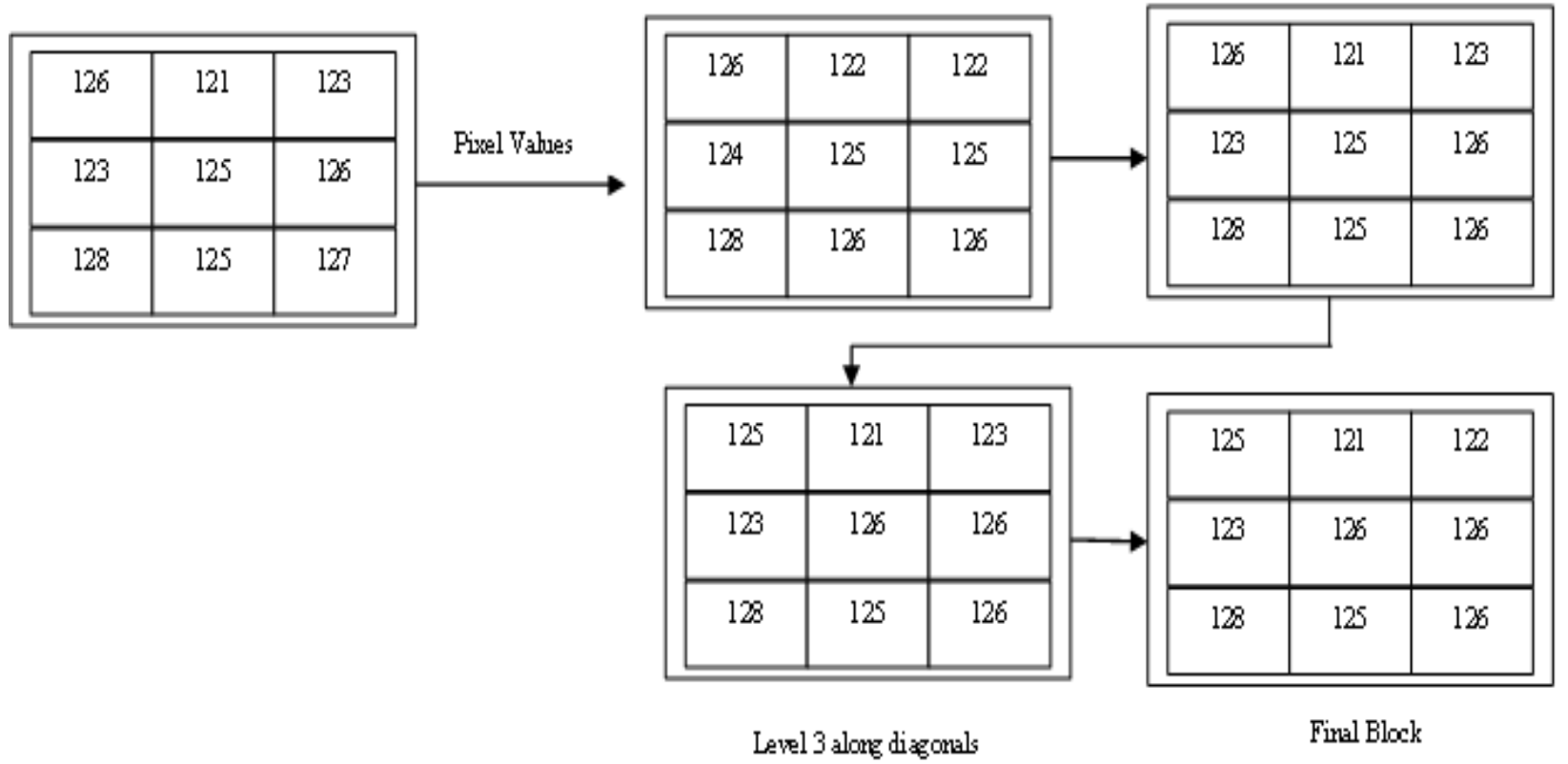

Figure 3 Example of the proposed 3-D compression technique

Level 1: The medical MRI brain image is given as an input to the encoder. It is divided into $8 \times 8$ blocks. For $i=0$ to 7 and $j=0$ to 7 identify the nearest neighbourhood of the pixels along the row. The distance offset $d_{\text {offset }}$ of the pixels are evaluated and the intensity of the pixels is assigned by taking the average between the pixel values.

$$
d_{\text {offset }}=\left|I_{B}-I_{A}\right|
$$

Where $I_{A}$ be the intensity of the pixel; $I_{B}$ be the intensity of the neighbouring pixel along rows and $d_{\text {offset }}$ depicts the distance offset. The intensity of the pixel is assigned based on

$$
\begin{aligned}
& I_{A}, I_{B}= \\
& \left\{\begin{array}{c}
\frac{I_{A}+I_{B}}{2} \\
I_{A}, \text { otherwise }
\end{array}, \text { for } d_{\text {offset }}<3\right.
\end{aligned}
$$

Level 2: For $i=0$ to 7 and $j=0$ to 7 identify the nearest neighbourhood of the pixels along the columns. The distance offset $d_{\text {offset }}$ of the pixels are evaluated and the intensity of the pixels is assigned by taking the average between the pixel values.

Level 3: For $i=0$ to 7 and $j=0$ to 7 identify the nearest neighbourhood of the pixels along the diagonals. The distance offset $d_{\text {offset }}$ of the pixels are evaluated and the intensity of the pixels is assigned by taking the average between the pixel values.
Thus the proposed compression method is evaluated using 3-Dimentionaly to de-correlate the pixel values. Figure 3 shows the example of the level 3-3-D compression method. From the figure it could be seen that initially the $3 \times 3$ image block contains pixels with nine different intensity values, whereas after applying the proposed 3-level 3-D image compression we got only 6 different intensity values. Such variation in intensity values gives compressed image with some degradation; which in turn is a visually lossless.

\subsection{Tri mode encoding scheme}

Histogram processing represents the probability of occurrence of particular intensity in an image. These histogram features are used in the proposed work in an efficient encoding. The feature used here is the number of occurrences of a particular intensity value. Based on that the encoding scheme is divided into three modes which is called the proposed tri-mode encoding scheme.

Mode 1: Low-level intensity is considered in mode 1. Number of occurrences less than a certain threshold value will be considered as low- level intensity. In the proposed work they are considered as nonimportant detailed image. Highest number of bits are assigned to those pixels, which reduces the entropy of the pixels.

Mode 2: High-level intensity is considered in mode 2. Number of occurrences greater than certain Mode 1 threshold value will be considered as high- level 


\section{J. Ashpin Pabi et al.}

intensity. In the proposed work they are considered as detailed image. Lowest number of bits are assigned to those pixels, which reduces the entropy of the pixels.

Mode 3: Frequently varying pixels are considered in mode 3 .
Number of occurrences that lie between low-level and high level threshold values will be considered as frequently varying pixels. In the proposed work, they are considered as frequently varying image and their bits are assigned by Huffman encoding.

Algorithm 1: Encoding Algorithm: Algorithm of encoding using the proposed 3-D compression technique

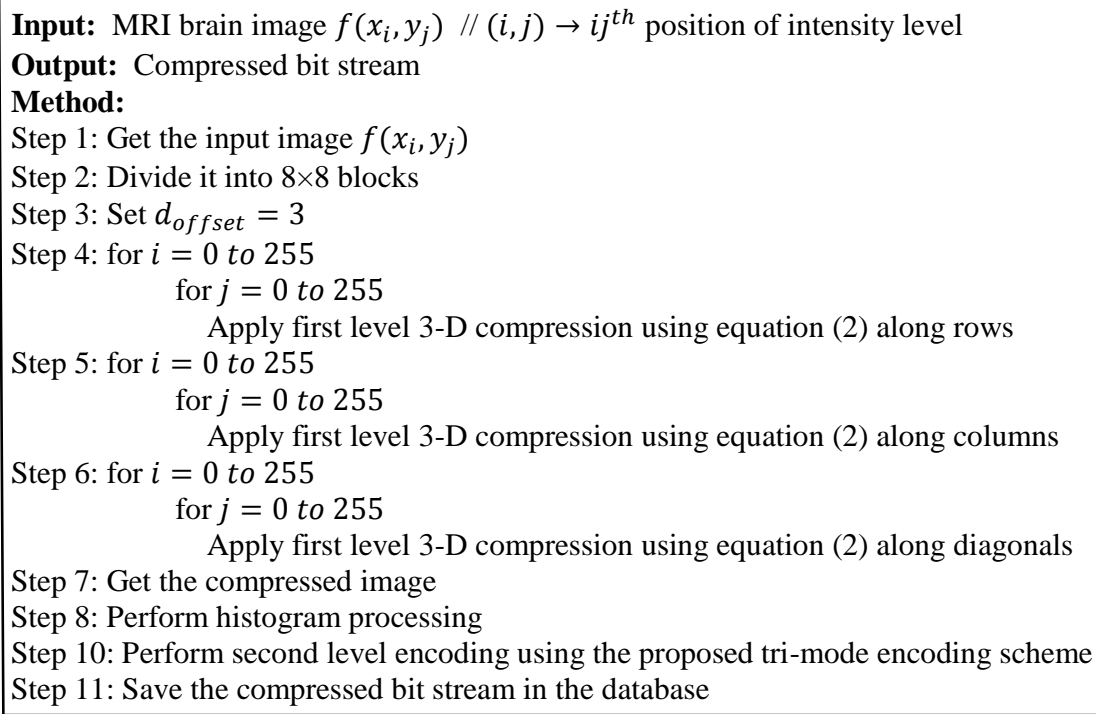

Algorithm 2: Decoding Algorithm: Algorithm of decoding using the proposed 3-D compression technique

Input: Compressed bit streams

Output: Reconstructed image

Method:

Step 1: Input compressed bit streams to the decoder

Step 2: Decode the compressed bit streams

Step 3: Apply inverse of the proposed 3-D compression

Step 4: Get the reconstructed images

In this section, we apply the proposed 3-D compression algorithm to several images. The test data comprises both MRI brain and standard images acquired from online databases includes OASIS (www.oasis-brain.org) and public-domain test images for homework and projects (http://homepages.cae.wisc.edu/ ece 533/images/) image databases respectively. To evaluate the performance, we compare the quality of the reconstructed image and the compression efficiency with SVD, WDR and JPEG. We consider peak signal-to-noise-ratio (PSNR) as an objective measure of the quality of the reconstructed image [12].

$$
P S N R=10 * \log \left(\frac{255^{2}}{M S E}\right)
$$

Mean Square Error (MSE) is calculated by

$M S E=\frac{1}{M N} \sum_{i=0}^{M} \sum_{j=0}^{N}(I(i, j)-J(i, j))^{2}$
Other metrics include:

compression ratio $(\mathrm{CR})=\frac{a}{b}$

Bits per piel (bpp) $=\frac{c}{n}$

Where a be the size of the uncompressed original image and $\mathrm{b}$ denotes the size of the compressed reconstructed image; $\mathrm{c}$ be the size of the compressed image in bits and $\mathrm{n}$ be the total number of pixels in bits.

The subjective results by SVD, WDR, JPEG and the proposed 3-D compression algorithm are almost same which is shown in Figure 4, but the proposed 3D compression method outperforms in terms of all the objective metrics, including PSNR, compression ratio and bits per pixel. 


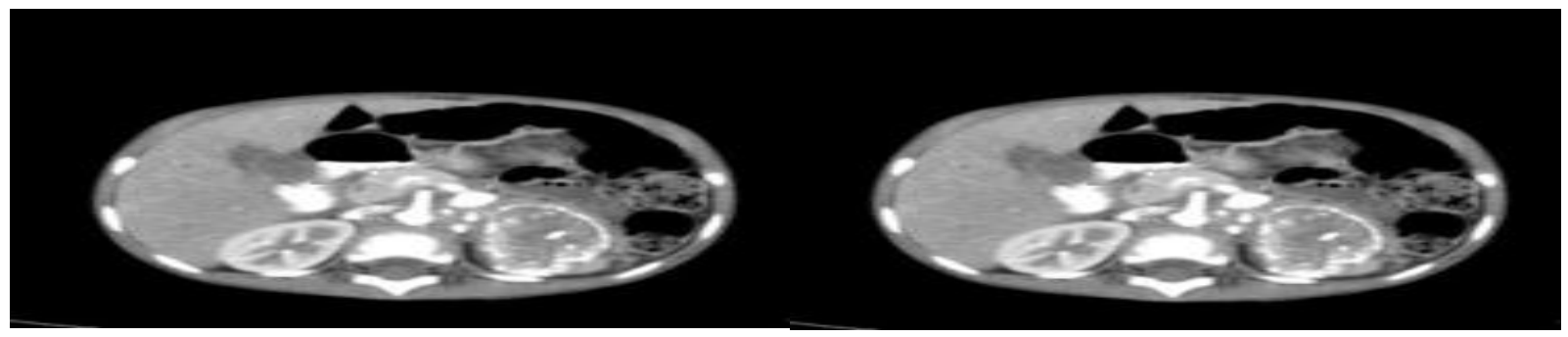

(a) Original Image

(b) SVD

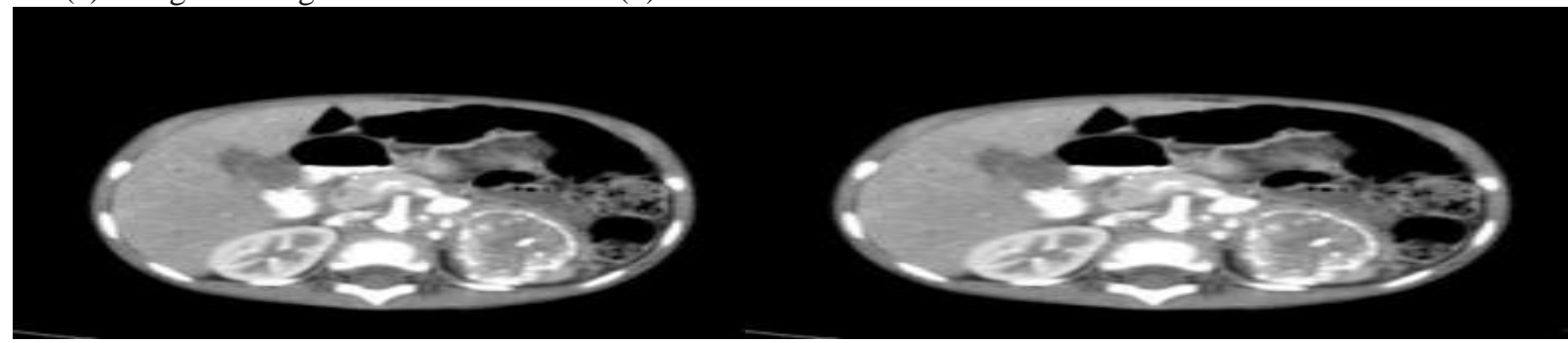

(c) WDR

(d) Proposed 3-D compression Method

Figure 4 Subjective valuation of the proposed 3-D compression method

Table 1 Objective evaluation of images

\begin{tabular}{llll}
\hline Method & Average PSNR & BPP & Compression ratio \\
\hline SVD & 33.54 & 0.23 & 6.57 \\
WDR & 35.43 & 0.98 & 8.36 \\
JPEG & 39.24 & 0.76 & 9.12 \\
Proposed 3-D compression & 40.22 & 0.16 & 14.04 \\
\hline
\end{tabular}

Table 2 Quality metrics of different images for the proposed 3-D compression method

\begin{tabular}{llll}
\hline Images & PSNR(DB) & CR & BPP \\
\hline & 40.23 & 14.34 & 0.19 \\
\hline & 39.34 & 13.37 & 0.16 \\
\hline & 41.34 & 14.45 & 0.15 \\
& 40.34 & 14.24 & 0.17 \\
\hline
\end{tabular}

The objective metrics are compared with the other existing methods in Table 1 in terms of average PSNR, compression ratio and bits-per pixel. The proposed 3-D compression method not only provides the better PSNR with $40.22 \mathrm{~dB}$ and the bpp of 0.16 , also gives the highest compression ratio of 14.04, which is higher than the other existing methods. The proposed compression algorithm is finally tested for standard images. The quality metrics of the proposed technique for those images are depicted in Table 2. The quality of the standard cameraman image is $39.34 \mathrm{~dB}$ with the CR 13.37 and the bpp 0.16.

\section{Conclusion}

In this paper, a 3-D image compression technique has been presented. We train with a variety of medical MRI images. Implementation of such intensity based 3-D compression increases the compression ratio of 
the image, also retains the quality of the reconstructed images. Experimental results showed that the proposed compression method provides efficient compression by maintaining the image quality. Experiments on color images will be done as a future work.

\section{Acknowledgment}

None.

\section{Conflicts of interest}

The authors have no conflicts of interest to declare.

\section{References}

[1] Moonen M, Van Dooren P, Vandewalle J. A singular value decomposition updating algorithm for subspace tracking. SIAM Journal on Matrix Analysis and Applications.1992; 13(4):1015-38.

[2] Kahu S, Rahate R. Image compression using singular value decomposition. International Journal of Advancements in Research \& Technology. 2013; 2(8):244-8.

[3] Konda T, Nakamura Y. A new algorithm for singular value decomposition and its parallelization. Parallel Computing. 2009;35(6):331-44.

[4] Andrews H, Patterson C. Singular value decompositions and digital image processing. IEEE Transactions on Acoustics, Speech, and Signal Processing. 1976; 24(1):26-53.

[5] Kamm JL. SVD-based methods for signal and image restoration (Doctoral dissertation, $\mathrm{PhD}$ thesis).1998.

[6] Yang JF, Lu CL. Combined techniques of singular value decomposition and vector quantization for image coding. IEEE Transactions on Image Processing. 1995;4(8):1141-6.

[7] $\mathrm{Hu}$ A, Zhang R, Yin D, Zhan Y. Image quality assessment using a SVD-based structural projection. Signal Processing: Image Communication. 2014;29(3):293-302.

[8] Gupta P, Purohit GN, Bansal V. A survey on image compression techniques. International Journal of Advanced Research in Computer and Communication Engineering. 2014;3(8):7762-8.

[9] Shih YT, Chien CS, Chuang CY. An adaptive parameterized block-based singular value decomposition for image de-noising and compression. Applied Mathematics and Computation. 2012;218(21):10370-85.

[10] Zhang YJ, Li SH, Wang SL. Detecting shifted double JPEG compression tampering utilizing both intrablock and inter-block correlations. Journal of Shanghai Jiaotong University (Science). 2013;18(1):716 .
[11] Rufai AM, Anbarjafari G, Demirel H. Lossy image compression using singular value decomposition and wavelet difference reduction. Digital Signal Processing. 2014;24:117-23.

[12] ZainEldin H, Elhosseini MA, Ali HA. Image compression algorithms in wireless multimedia sensor networks: a survey. Ain Shams Engineering Journal. 2015;6(2):481-90.

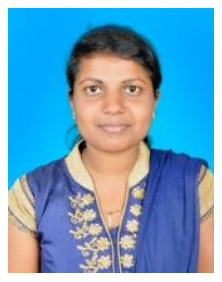

D. J. Ashpin Pabi was born in 1990 in India. She received her B.Tech degree in Information and Communication Technology from Manonmaniam Sundaranar University, Thirunelveli in 2011 and M.E in software Engineering from Vins Christian College of Engineering, Nagerkoil in 2013. She is currently a Research Scholar in Annamalai University, Chidambaram. Her main research focus is on Image Processing.

Email: ashpinpabi@gmail.com

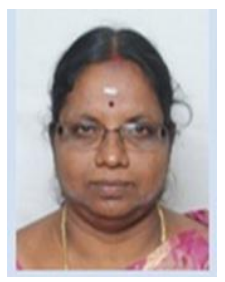

Dr. P. Aruna was born in 1968 in India. She received her B.E. from Madras University, M. Tech from IIT Delhi and the Ph.D degree from Annamalai University. Presently she is working as a Professor in the Department of Computer Science and Engineering of Annamalai University. She has published 80 research papers in international journals and conferences and 26 research papers in national journals and conferences. She has twenty five years of teaching experience and sixteen years of research experience. She has published 3 book chapters. Her area of specialization includes Neural Networks \& Fuzzy Systems, Data Mining and Image processing. She has guided 6 Ph.D scholars.

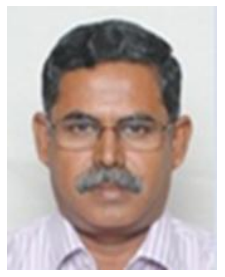

N. Puviarasan was born in 1963 in India. He received his M.S (Soft Sys) from BITS, Pilani, M.S (Engg) from Annamalai University. Presently he is working as an Associate Professor in the Department of Computer Science and Engineering of Annamalai University. $\mathrm{He}$ has published 25 research papers in international journals and conferences and 13 research papers in national journals and conferences. He has twenty seven years of teaching experience and thirteen years of research experience. He has published one book chapter. His area of specialization includes Neural networks and Fuzzy systems, Data mining and Image Processing. 